\title{
THE ISOPERIMETRIC PROBLEM OF THE CONVEX HULL OF A CLOSED SPACE CURVE
}

\author{
Z. A. MELZAK
}

I. Let $C$ be a closed rectifiable curve or an open rectifiable arc in the $n$-dimensional Euclidean space $E_{n}$. Let $\bar{C}, V(\bar{C})$ and $L(C)$ denote respectively the closed convex hull of $C$, the volume of $\bar{C}$ and the length of $C$. Let $(c, n)$, or $(0, n)$, stand for the problem of maximizing $V(\bar{C})$, subject to the condition $L(C)=$ const, for a closed curve $C$, or an open arc $C$. It is clear that the solution is to be given only up to a similarity and a rigid motion, possibly followed by a reflexion.

On closer examination it turns out that there are four classes of problems: $(c, 2 n),(0,2 n),(0,2 n+1)$ and $(c, 2 n+1)$. Under certain restrictive assumptions $(c, 2 n)$ has been solved by Schoenberg [1]. Generalizing the Fourier-series isoperimetric method of Hurwitz [2], he proves that the solution $C$ of $(c, 2 n)$ is given parametrically by

$$
\begin{aligned}
& x_{1}(t)=\sin t, \quad x_{3}(t)=\frac{1}{2} \sin 2 t, \quad x_{5}(t)=\frac{1}{3} \sin 3 t, \cdots \\
& x_{2}(t)=\cos t, \quad x_{4}(t)=\frac{1}{2} \cos 2 t, \quad x_{6}(t)=\frac{1}{3} \cos 3 t, \cdots
\end{aligned}
$$

with $0 \leqq t<2 \pi$. The corresponding isoperimetric inequality is

$$
V(\bar{C}) \leqq\left[(2 \pi n)^{n}(2 n) ! n !\right]^{-1} L^{2 n}(C) .
$$

On the basis of the analogy between (1) and the isoperimetric problems of the circle and the semicircle it is likely that the solution $C$ of $(0,2 n)$ is an open arc, also given by (1), but with $0 \leqq t \leqq \pi$. The isoperimetric inequality would then be

$$
V(\bar{C}) \leqq 2^{n-1}\left[(\pi n)^{n}(2 n) ! n !\right]^{-1} L^{2 n}(C) .
$$

The case $(0,3)$ has been treated by Egervary [3], who proves that here the solution is one turn of the circular helix

$$
x(t)=\sin t, \quad y(t)=\cos t, \quad z(t)=2^{-1 / 2} t,
$$

and the isoperimetric inequality is

$$
V(\bar{C}) \leqq\left(18.3^{1 / 2} \pi\right)^{-1} L^{3}(C) .
$$

Presented to the Society, April 6, 1957 under the title Convex hulls of a class of closed space curves. Preliminary report; received by the editors December 6, 1957 and, in revised form, January 10, 1959.

1 The author is now with the Bell Telephone Laboratories, Inc., Murray Hill, N. J. 
Egervary's method carries over to the general case $(0,2 n+1)$; the solution is then a higher-dimensional analog of a turn of a circular helix.

The last class of problems, $(c, 2 n+1)$, does not seem to have been treated so far, although $(c, 3)$ was proposed by Bonnesen and Fenchel in 1934, [4, p. 111]. Here $(c, 3)$ is considered and a partial solution is given.

II. From now on a curve $C$, unless otherwise defined, will mean a closed rectifiable curve in $E_{3} . C$ is said to be smooth if along $C$ the Cartesian coordinates $x(s), y(s)$ and $z(s)$ are continuously differentiable functions of the arc-length $s$. A curve $C$ belongs to the class I if

(2) $C$ is smooth,

(3) $C$ has two orthogonal planes of symmetry $P_{1}$ and $P_{2}$,

(4) $C$ projects on $P_{1}$, and on $P_{2}$, as a convex open arc, and on $P_{3}$ (a plane orthogonal to $P_{1}$ and $P_{2}$ ) as a closed convex plane curve.

Geometrically interpreted, these conditions state that $C$ is the intersection of certain two perpendicular smooth convex symmetric cylinders. We can now select a Cartesian coordinate system so that

$$
\begin{aligned}
& x(0)>0, \quad y(0)=0, \quad z(0)=0, \\
& x^{\prime}(0)=0, \quad z^{\prime}(0)=0, \quad z(s) \geqq 0,
\end{aligned}
$$

and

$$
\begin{gathered}
x^{\prime}(s) \leqq 0, \quad y^{\prime}(s) \geqq 0, \quad z^{\prime}(s) \geqq 0, \\
0 \leqq s \leqq L / 4, \quad L=L(C) .
\end{gathered}
$$

Here and elsewhere accent denotes differentiation with respect to the arc-length $s$. The symmetry condition implies that $C$ cuts $P_{1}$ and $P_{2}$ at right angles:

$$
\begin{aligned}
x^{\prime}(0) & =z^{\prime}(0)=0, & y^{\prime}(0) & =1 ; \\
x^{\prime}(L / 2) & =z^{\prime}(L / 2)=0, & y^{\prime}(L / 2) & =-1 ; \\
x^{\prime}(L / 4) & =-1, & y^{\prime}(L / 4) & =z^{\prime}(L / 4)=0 ; \\
x^{\prime}(3 L / 4) & =1, & y^{\prime}(3 L / 4) & =z^{\prime}(3 L / 4)=0 .
\end{aligned}
$$

Lemma 1. If $C \in \mathfrak{A}$ then

$$
V(\bar{C})=\int_{0}^{L} x y z^{\prime} d s .
$$

By the conditions (2)-(4) a plane $z=z_{0}, 0 \leqq z_{0} \leqq z(L / 4)$, cuts $C$ in 
four vertices of a rectangle; let $K$ be the union of all such rectangles. Then $K$ is a convex solid containing $\bar{C}$ and through any point of the boundary of $K$ there passes a straight segment with end-points on $C$. Therefore $\bar{C}=K$. Let $p_{1}$ and $p_{2}$ be two points on $C$, both in the same octant, and let $H_{1}$ and $H_{2}$ be the horizontal quarter-planes through them. That is, $H_{i}$ is the part of the horizontal plane through $p_{i}$ in the octant of $p_{i}$. Taking now the largest parallelepiped between $H_{1}$ and $H_{2}$ in $\bar{C}$ as the volume element, and performing the usual operations of setting up a definite integral, we obtain (8). It will be noticed that in each of the four octants of $C$ either $x, y$ and $z^{\prime}$ are all nonnegative, or two of them are nonpositive, so that the integrand in (8) is always nonnegative.

Lemma 2. Let

$$
\begin{array}{ccc}
V=\int_{0}^{L} x y z^{\prime} d s, & x^{\prime 2}+y^{\prime 2}+z^{\prime 2}-1=0, & x^{\prime}(0)=y(0)=z^{\prime}(0)=0, \\
x(0)=x(L), & y(0)=y(L), & z(0)=z(L) .
\end{array}
$$

Then a necessary condition for $V$ to be maximum is that the following system of differential equations be satisfied:

$$
k^{2} x^{\prime \prime}=-x y^{2}, \quad k^{2} y^{\prime \prime}=-y x^{2}, \quad k z^{\prime}=x y, \quad k \text { a constant } .
$$

By the theory of the Lagrange multipliers [5], the above problem reduces to that of maximizing the single integral

$$
\begin{aligned}
J & =\int_{0}^{L} F\left(s, x, y, z, x^{\prime}, y^{\prime}, z^{\prime}\right) d s \\
& =\int_{0}^{L}\left[x y z^{\prime}-\lambda\left(x^{\prime 2}+y^{\prime 2}+z^{\prime 2}-1\right)\right] d s,
\end{aligned}
$$

where $\lambda=\lambda(s)$ is the multiplier. The Euler-Lagrange equations for $J$ are

$$
\left(2 \lambda x^{\prime}\right)^{\prime}=-y z^{\prime}, \quad\left(2 \lambda y^{\prime}\right)^{\prime}=-x z^{\prime}, \quad\left(x y-2 \lambda z^{\prime}\right)^{\prime}=0 .
$$

In view of the assumed initial conditions and transversality, the last equation yields

$$
x y=2 \lambda z^{\prime} .
$$

If this is used to eliminate $z^{\prime}$ from (10), one obtains

$$
2 \lambda\left(2 \lambda x^{\prime}\right)^{\prime}=-x y^{2}, \quad 2 \lambda\left(2 \lambda y^{\prime}\right)^{\prime}=-y x^{2} .
$$

Multiplying the first equation by $x^{\prime}$, the second one by $y^{\prime}$, and adding 
the results, we have

$$
\left[4 \lambda^{2}\left(x^{\prime 2}+y^{\prime 2}\right)+x^{2} y^{2}\right]^{\prime}=0,
$$

and by the initial conditions

$$
4 \lambda^{2}\left(x^{\prime 2}+y^{\prime 2}\right)+x^{2} y^{2}=k^{2}, \quad k \text { a constant. }
$$

Comparing (11), (12) and the arc-length condition $x^{\prime 2}+y^{\prime 2}+z^{\prime 2}-1$ $=0$, we get $k^{2}=4 \lambda^{2}$ and the lemma follows.

Lemma 3. The necessary condition of Lemma 2 is also sufficient.

Without loss of generality let $\lambda=k / 2>0$. We form first the Weierstrass $E$-function and the Legendre quadratic form $\Omega$ for the integral $T$ in (9). These are defined as follows, [5]:

$$
\begin{aligned}
E\left(s, x, y, z, x^{\prime}, y^{\prime}, z^{\prime}, p, q, r\right)= & F(s, x, y, z, p, q, r)-F\left(s, x, y, z, x^{\prime}, y^{\prime}, z^{\prime}\right) \\
& -\left(p-x^{\prime}\right) F_{x^{\prime}}\left(s, x, y, z, x^{\prime}, y^{\prime}, z^{\prime}\right) \\
& -\left(q-y^{\prime}\right) F_{y^{\prime}}\left(s, x, y, z, x^{\prime}, y^{\prime}, z^{\prime}\right) \\
& -\left(r-z^{\prime}\right) F_{z^{\prime}}\left(s, x, y, z, x^{\prime}, y^{\prime}, z^{\prime}\right), \\
\Omega\left(\xi_{1}, \xi_{2}, \xi_{3}\right)= & \sum_{i, j=1}^{s} F_{x_{j}^{\prime} x_{j}^{\prime}}\left(s, x, y, z, x^{\prime}, y^{\prime}, z^{\prime}\right) \xi_{i} \xi_{j},
\end{aligned}
$$

where $x_{1}=x, x_{2}=y, x_{3}=z$. Performing the calculations, we have

$$
\begin{aligned}
& E=-\lambda\left[\left(p-x^{\prime}\right)^{2}+\left(q-y^{\prime}\right)^{2}+\left(r-z^{\prime}\right)^{2}\right], \\
& \Omega=-2 \lambda\left(\xi_{1}^{2}+\xi_{2}^{2}+\xi_{3}^{2}\right) .
\end{aligned}
$$

Thus two conditions for a relative strong maximum are satisfied. The remaining two conditions are also satisfied, and by the suffciency theorem for Bolza's problem, [5, p. 235], the lemma follows.

III. In this section we shall investigate the differential equations describing $C$. By a change of scale it will be assumed that $k=1$, and the equations become $x^{\prime \prime}=-x y^{2}, y^{\prime \prime}=-y x^{2}, z^{\prime}=x y$.

Lemma 4. The differential system

$$
x^{\prime \prime}=-x y^{2}, \quad y^{\prime \prime}=-y x^{2},
$$

possesses a unique solution $x(s), y(s)$, which is analytic for all real $s$, for any initial conditions $x(0)=a_{0}, x^{\prime}(0)=a_{1}, y(0)=b_{0}, y^{\prime}(0)=b_{1}$.

As in (12), we show that there exists a first integral

$$
x^{\prime 2}+y^{\prime 2}+x^{2} y^{2}=a_{1}^{2}+b_{1}^{2}+a_{0}^{2} b_{0}^{2}=c^{2} . \quad c>0 .
$$


This implies at once the following estimates:

$$
\left|x^{\prime}\right| \leqq c, \quad\left|y^{\prime}\right| \leqq c,
$$

and therefore also

$$
|x(s)| \leqq c_{0}+c|s|, \quad|y(s)| \leqq c_{0}+c|s|,
$$

where $c_{0}=\max \left(\left|a_{0}\right|,\left|b_{0}\right|\right)$. We show first that if $x\left(s_{0}\right)$ and $y\left(s_{0}\right)$ are finite for some $s_{0}$, then $x(s)$ and $y(s)$ are analytic for $\left|s-s_{0}\right|$ $<\min \left(1,1 / c, 1 /\left|x\left(s_{0}\right)\right|, 1 /\left|y\left(s_{0}\right)\right|\right)$. Assume that

$$
x(s)=\sum_{0}^{\infty} \alpha_{n}\left(s-s_{0}\right)^{n}, \quad y(s)=\sum_{0}^{\infty} \beta_{n}\left(s-s_{0}\right)^{n} ;
$$

substituting into (13) and comparing coefficients, we have

$$
\begin{array}{ll}
(n+2)(n+1) \alpha_{n+2} & =-\sum_{i+j+k=n} \alpha_{i} \beta_{j} \beta_{k}, \\
(n+2)(n+1) \beta_{n+2} & =-\sum_{i+j+k=n} \beta_{i} \alpha_{j} \alpha_{k},
\end{array}
$$

Let $R=\max \left(1, c,\left|\alpha_{0}\right|,\left|\beta_{0}\right|\right)$, so that in view of (15)

$$
\left|\alpha_{j}\right| \leqq R^{j+1}, \quad\left|\beta_{j}\right| \leqq R^{j+1}, \quad j=0,1 .
$$

Assume that (19) holds for $j \leqq n+1$. Then by (18)

$$
(n+2)(n+1)\left|\alpha_{n+2}\right| \leqq R^{n+3} \sum_{i+j+k=n} 1=\frac{1}{2}(n+2)(n+1) R^{n+3},
$$

and similar estimate holds for $\left|\beta_{n+2}\right|$. It follows that (19) holds for $j=n+2$ and, by induction on $n$, for all $j$. Therefore the power series (17) converge for $\left|s-s_{0}\right|<1 / R=\min \left(1,1 / c, 1 /\left|x\left(s_{0}\right)\right|, 1 /\left|y\left(s_{0}\right)\right|\right)$, which was the assertion.

In particular, by putting $s_{0}=0$ it follows that $x(s)$ and $y(s)$ are analytic for $0 \leqq s<\min \left(1,1 / c, 1 /\left|a_{0}\right|, 1 /\left|b_{0}\right|\right)=s_{1}$. Now we repeat the same procedure with $s_{1}-\epsilon_{1}$ in place of $s_{0}$. Here $\epsilon_{1}$, and later $\epsilon_{n}$, is a small positive constant. The result is that $x(s)$ and $y(s)$ remain analy tic for $s_{1}-\epsilon_{1} \leqq s<s_{2}$, where $s_{2}=\min \left(1,1 / c, 1 /\left|x\left(s_{1}-\epsilon_{1}\right)\right|, 1 /\left|y\left(s_{1}-\epsilon_{1}\right)\right|\right)$. By (16) $\left|x\left(s_{1}-\epsilon_{1}\right)\right| \leqq c_{0}+c s_{1}$ and $\left|y\left(s_{1}-\epsilon_{1}\right)\right| \leqq c_{0}+c s_{1}$, so that $s_{2}$ $\geqq \min \left(1,1 / c, 1 /\left(c_{0}+c s_{1}\right)\right)$. If this process is continued, we obtain a sequence of analytic continuation intervals: $0 \leqq s<s_{1}, s_{1}-\epsilon_{1} \leqq s$ $<s_{2}, \cdots, s_{n}-\epsilon_{n} \leqq s<s_{n+1}$, where by (16)

$$
s_{n+1} \geqq \min \left(1,1 / c, 1 /\left(c_{0}+c s_{n}\right)\right)>0 .
$$

Thus in $n$ steps $x(s)$ and $y(s)$ are shown to be analytic for $s$ in the 
interval $0 \leqq s \leqq \sum_{1}^{n} s_{j}-\sum_{1}^{n} \epsilon_{j}$. The estimate (20) implies at once that $\sum_{1}^{n} s_{j}$ diverges with $n$. Since the sum $\sum_{1}^{n} \epsilon_{j}$ can be made uniformly small, it follows that $x(s)$ and $y(s)$ are analytic for $s \geqq 0$. Their analyticity for $s \leqq 0$ can be shown in the same way. Since the constructed analytic solutions are clearly unique, the lemma is proved.

For our purposes we shall consider only the initial conditions of the form $x(0)=a>0, x^{\prime}(0)=y(0)=0, y^{\prime}(0)=b>0$. The graph of the solution $x(s), y(s)$ with these initial data, which exists and is unique by Lemma 4 , will be denoted by $D(a, b)$. A subgraph corresponding to $0 \leqq s \leqq s_{1}$, will be denoted by $D\left(a, b, s_{1}\right)$. In the $x y$-plane of the graph let $Q$ be the first quadrant and $B$ that part of the diagonal $x=y$ which is in $Q . D_{Q}\left(a, b, s_{1}\right)$ is defined by: (1) $D_{Q}\left(a, b, s_{1}\right) \subset Q$, (2) if $D\left(a, b, s_{2}\right)$ $\subset Q$ then $D\left(a, b, s_{2}\right) \subset D_{Q}\left(a, b, s_{1}\right)$.

Lemma 5. If $y^{\prime} \geqq 0$ along $D(a, b, s) \subset Q$, then along $D(a, b, s)$

$$
\begin{aligned}
& y^{\prime 2}+a^{2} y^{2}-b^{2} \geqq 0, \\
& y(s) \geqq b / a \sin a s,
\end{aligned}
$$

and $D(a, b, s)$ itself is a convex arc.

Since $y^{\prime}(0)=b>0, y^{\prime} \geqq 0$ initially for small $s$ along any $D\left(a, b, s_{1}\right)$. By (13) $x^{\prime \prime}<0$ inside $Q$ and $x^{\prime}(0)=0$, so that $x(s)$ is a decreasing function in $Q: x(s) \leqq x(0)=a$. Now by (13) $y^{\prime \prime} \geqq-a^{2} y$. By the hypothesis $y^{\prime} \geqq 0$ so that $y^{\prime \prime} y^{\prime} \geqq-a^{2} y y^{\prime}$; the last relation can be written as $\left(y^{\prime 2}+a^{2} y^{2}-b^{2}\right)^{\prime} \geqq 0$ and (21) follows by integrating. (22) is a simple consequence of (21) and the assumption $y^{\prime} \geqq 0$, and is obtainable similarly. To prove the convexity of $D(a, b, s)$ we observe that the second derivative $d^{2} y / d x^{2}=\left(x^{\prime} y^{\prime \prime}-x^{\prime \prime} y^{\prime}\right) / x^{\prime 3}$ cannot change its sign along $D(a, b, s)$ because on the interior of $D(a, b, s) x^{\prime}, x^{\prime \prime}$ and $y^{\prime}$ are negative while $y^{\prime} \geqq 0$.

If $D_{Q}\left(a, b, s_{1}\right)$ cuts $B$, let $s_{m}$ be the smallest value of $s$ for which this happens, and let $\gamma$ be the corresponding angle at $s_{m}$. To fix $\gamma$ uniquely we take along $D_{Q}$ the direction of increasing $s$ and along $B$ the direction away from the origin, counting the angle positive.

Lemma 6. If $a>0$ is fixed and $b \geqq a^{2}$, then $D_{Q}\left(a, b, s_{1}\right)$ cuts $B$ and

$$
\begin{aligned}
& \gamma=\gamma(b)>\pi / 4, \\
& \lim _{b \rightarrow \infty} \gamma(b)=\pi / 4 .
\end{aligned}
$$

If $b \leqq a^{2} / 4$, then either $D_{Q}\left(a, b, s_{1}\right)$ does not intersect $B$, or if it does,

$$
\gamma(b)>\pi / 2 \text {. }
$$


Let $a>0$ be fixed and consider $D=D_{\mathbf{Q}}\left(a, b, s_{1}\right)$. If $y^{\prime}$ vanishes along $D$, say for $s=s_{2}$, then by (21) $y\left(s_{2}\right) \geqq b / a$. Therefore, if $b \geqq a^{2}$, then $y\left(s_{2}\right) \geqq a$ while $x\left(s_{2}\right) \leqq x(0)=a$, so that $D$ must cut $B$ for some $s_{3}$, $0<s_{3} \leqq s_{2}$. Suppose now that $D$ lies below $B$ and $y^{\prime}>0$ along $D$. This may occur only if $s_{1}=\infty$. However, by putting $s=\pi / 2 a$ in (22) we have now $y(\pi / 2 a) \geqq b / a$ and if $b \geqq a^{2}$ this leads to a contradiction: $D$ cannot lie below $B$. This establishes the first part of the lemma; (23) and (24) are obvious conclusions since $x$ and $y^{\prime}$ are steadily decreasing.

From the first integral (14) we have $x^{\prime 2}+y^{\prime 2}+x^{2} y^{2}=b^{2}$ for all $s$. Therefore $D$ cannot pass in $Q$ above the hyperbola $x y=b$, and the last conclusion of the lemma follows now from the geometry of the situation.

LEMMA 7. If $\gamma=\gamma(b)$ is defined at $b=b_{1}$ and $\gamma\left(b_{1}\right) \neq \pi$, then $\gamma(b)$ is also defined over some interval $\left(b_{1}-\epsilon, b_{1}+\epsilon\right), \epsilon>0$. If $\gamma(b)$ is defined for all $b \epsilon\left(b_{1}, b_{2}\right)$ and $\gamma(b)<m<\pi$, then $\gamma\left(b_{1}\right)$ and $\gamma\left(b_{2}\right)$ exist and $\gamma(b)$ is continuous over $\left[b_{1}, b_{2}\right]$.

To bring out the dependence on the initial conditions, we shall write, where necessary, $x(a, b, s)$ and $y(a, b, s)$ for $x(s)$ and $y(s)$. The proof of the lemma follows simply from the following three observations. In the first place, to fix the value of $s$ at which $D_{Q}\left(a, b, s_{1}\right)$ cuts $B$, we have the equation $x(a, b, s)=y(a, b, s)$. By the implicit function theorem $s=s(b)$ is then a continuous solution unless the Jacobian condition fails, i.e., unless $x^{\prime}(a, b, s)=y^{\prime}(a, b, s)$, that is, unless $D_{Q}$ is tangent to $B$ and $\gamma=\pi$. In the second place, by the fundamental theorems for differential equations [6, Chapter 1] $x(a, b, s), x^{\prime}(a, b, s)$, $y(a, b, s)$ and $y^{\prime}(a, b, s)$ are continuous in $a, b$ and $s$ jointly. Finally,

$$
\begin{aligned}
\gamma(b)= & \arctan \left\{\left[y^{\prime}(a, b, s(b))-x^{\prime}(a, b, s(b))\right]\right. \\
& \left.\div\left[y^{\prime}(a, b, s(b))+x^{\prime}(a, b, s(b))\right]\right\},
\end{aligned}
$$

so that $\gamma(b)$ depends continuously on $x^{\prime}(a, b, s(b))$ and $y^{\prime}(a, b, s(b))$, and so on $b$.

Lemma 8. For each $a>0$ there exists a value $b=b(a)$, such that $D_{Q}\left(a, b(a), s_{1}\right)$ cuts $B$ and $\gamma[b(a)]=\pi / 2$.

Let $a>0$ be fixed. By Lemma $6, \gamma(b)$ is defined for all $b \geqq a^{2}$ and $\gamma(M)<\pi / 4+1 / 10$ for $M$ large enough. Consider now the interval $I=\left[a^{2} / 4, M\right]$. If $\gamma(b)$ is defined over the whole of $I$, then by Lemmas 6 and $7 \gamma(b)=\pi / 2$ for some $b, a^{2} / 4<b<M$. If $\gamma(b)$ is not defined for all $b \in I$, there is a value $b_{1}, a^{2} / 4<b_{1}<M$, such that $\gamma(b)$ is defined for all $b \in\left(b_{1}, M\right]$, but not for all $b \epsilon\left[b_{1}-\epsilon, b_{1}\right]$ for any $\epsilon>0$. Assume 
that $\gamma(b)<\pi / 2$ for $b \epsilon\left(b_{1}, M\right]$. Then by Lemma $7 \gamma\left(b_{1}\right)$ exists and $\gamma(b)$ is continuous over $\left[b_{1}, M\right]$. If $\gamma\left(b_{1}\right)=\pi / 2$, the conclusion follows, suppose therefore that $\gamma\left(b_{1}\right)<\pi / 2$, so that $\gamma(b)<\pi / 2$ for $b \epsilon\left[b_{1}, M\right]$. Now Lemma 7 implies that $\gamma(b)$ can be continuously extended to a larger interval $\left[b_{1}-\epsilon, M\right], \epsilon>0$, which is a contradiction. Therefore $\gamma(b) \geqq \pi / 2$ for some $b \epsilon\left(b_{1}, M\right]$ and the conclusion follows as before.

Lemma 9. $D(a, b(a))$ is a closed convex curve with four axes of symmetry: $x=0, y=0, y= \pm x$.

By Lemma 8 there is an $\operatorname{arc} D=D\left(a, b(a), s_{1}\right)$ which starts orthogonally to the $x$-axis and terminates orthogonally to $B$ at $B$. It is clear that $y^{\prime}>0$ along $D$ so that by Lemma $5 D$ is a convex arc. Since the system (13) is formally invariant under the transformations $x \rightarrow y, y \rightarrow x$, it follows that the continuation of $D$ to the region in $\phi$ above $B$ is the reflexion $D_{1}$ of $D$ in $B$. Let $D_{2}=D \cup D_{1}$. The system (13) is also invariant under the transformations $x \rightarrow y, y \rightarrow-x$; this implies, as before, that the continuation $D_{3}$ of $D_{2}$ to the second quadrant is the reflexion of $D_{2}$ in the $y$-axis. Continuing in this way, we show that $D(a, b(a))$ must close up and be a convex curve with the required symmetries.

In view of the Lemmas 1-9 we have:

Theorem 1. Let $C \in \mathfrak{A}$ and let $L(C)$ be fixed. Then $V(\bar{C})$ is maximm if and only if $C$ is similar to a periodic solution of the system

$$
x^{\prime \prime}=-x y^{2}, \quad y^{\prime \prime}=-y x^{2}, \quad z^{\prime}=x y .
$$

IV. It will be indicated now how some restrictions on $C$ in Theorem 1 can be relaxed. Instead of assuming that $C$ is smooth it suffices to assume piecewise smoothness. The full condition follows: at the four points where $C$ cuts its symmetry planes by transversality, elsewhere by the Weierstrass-Erdmann corner condition, [5]. We define now the class $\mathfrak{B}$ of bicylindric curves, which is wider than $\mathfrak{A}$. Let $Z$ be a cylinder whose directrix is a simple plane open arc lying on one side of the straight line $L$ through its end-points. Let $P_{1}(Z)$ be the plane through $L$ parallel to the direction of $Z$, and let $P_{2}(Z)$ be the plane parallel to $P_{1}(Z)$ which supports $Z$ from the other side. A rectifiable curve is bicylindric if $C=Z_{1} \cap Z_{2}$, where $Z_{1}$ and $Z_{2}$ are two cylinders as described above, and $P_{1}\left(Z_{1}\right)=P_{2}\left(Z_{2}\right), P_{2}\left(Z_{1}\right)=P_{1}\left(Z_{2}\right)$. A curve $C$ in $\mathfrak{B}$ is called deficient if for some other curve $C_{1}$ in $\mathfrak{B}$

$$
V\left(\bar{C}_{1}\right) L^{3}(C)>V(\bar{C}) L^{3}\left(C_{1}\right) .
$$

It is clear that in solving $(c, 3)$ for curves in $\mathfrak{B}$ it suffices to consider 
nondeficient curves only. However, by simple symmetrization techniques, similar to those of Steiner [7], it may be shown that $C \in \mathfrak{B}$ is deficient unless $C \in \mathfrak{A}$. Furthermore, it appears likely that a single condition on $C$ in Theorem 1 would suffice: no plane cuts $C$ in more than four points.

V. Some problems related to $(c, 3)$ will now be listed. Is Theorem 1 valid under minimal restrictions on $C$ (rectifiability, being a closed curve)? Is the periodic solution of Theorem 1 unique? Next, $(c, 3)$ may be proposed with certain restrictions: $C$ lies on a sphere, or on a circular cylinder, or $C$ is topologically a prescribed type of knot.

Further problems may be mentioned in connection with the system (13). Can the method used here be applied to show that some other plane autonomous systems, of order two or more, have periodic solutions? Are there some other Hamiltonian systems of the form $f_{i}\left(x, x^{\prime}, \cdots, y, y^{\prime}, \cdots\right)=0, i=1,2$, which are "projections" of systems of the form $g_{i}\left(x, x^{\prime}, \cdots, y, y^{\prime}, \cdots, z, z^{\prime}, \cdots\right)=0, i=1,2,3$ in the same way in which (13) is "projection" of (10)? If so, when does the "original" system $g_{i}=0, i=1,2,3$, form the Euler-Lagrange equations of a variational problem in which a simple quantity associated with a closed space curve, as here the volume of the convex hull, is to be maximized?

Is there a simple procedure to calculate with arbitrary accuracy the isoperimetric constant of $(c, 3)$ through Theorem 1 ?

VI. $(c, 3)$ was suggested to the author by Professor I. J. Schoenberg, of the University of Pennsylvania, who also supplied most of the information in §I. Professor B. A. Rattray, of McGill University, made valuable suggestions in connection with Lemmas 6-8. The Editor has contributed suggestions for general improvement.

The work reported upon was started at the Massachusetts Institute of Technology in Summer 1956 with the support of the Air Force Office of Scientific Research, and continued in 1957 at the University of Michigan on a Research Associateship of the Office of Naval Research.

The help of these persons and institutions is gratefully acknowledged.

\section{BIBLIOGRAPHY}

1. I. J. Schoenberg, An isoperimetric inequality, Acta Math. vol. 91 (1954) pp. 143-164.

2. A. Hurwitz, Sur quelques applications geometriques, Ann. Ecole Norm. Sup. (3) vol. 19 (1902). 
3. E. Egervary, On the smallest convex cover, Publ. Math. Debrecen vol. 1 (1949) pp. 65-70.

4. T. Bonnesen and W. Fenchel, Theorie der konvexen Koerper, Ergebnisse der Math. vol. 1, 1934.

5. G. A. Bliss, Lectures on the calculus of variations, University of Chicago Press, 1946.

6. E. A. Coddington and N. Levinson, Theory of ordinary differential equations, McGraw-Hill, 1955.

7. W. Blaschke, Kreis und Kugel, Chelsea, 1949.

MCGill UNiversity

\section{EXTENDING DIFFEOMORPHISMS}

RICHARD S. PALAIS

In [ 1 , Theorem 5.5], the author proved the following fact. Let $M$ be a differentiable manifold, $p \in M$, and $f$ a diffeomorphism of a neighborhood of $p$ into $M$. If $M$ is orientable assume in addition that $f$ is orientation preserving. Then there exists a diffeomorphism of $M$ onto itself which agrees with $f$ in a neighborhood of $p$. In this paper we shall answer affirmatively a question raised by A. M. Gleason; namely whether the 0 -cell $p$ can be replaced by a differentiable $k$-cell. It turns out that this extension follows rather easily from the special case. The author understands that the theorem has been proved independently by J. Cerf in his thesis (not yet published). The author would like to thank Dr. S. Smale for several suggestions utilized in this paper.

1. Notation. $M$ will denote an $n$-dimensional differentiable $\left(=C^{\infty}\right)$ manifold. For $t=\left(t_{1}, \cdots, t_{k}\right) \in R^{k}$ we write $\|t\|^{2}=\sum_{i=1}^{k} t_{i}^{2}$. We put $B_{r}^{k}=\left\{t \in R^{k} \mid\|t\| \leqq r\right\}$ and $\dot{B}_{r}^{k}$ for the interior of $B_{r}^{k}$ in $R^{k}$. We regard $B_{r}^{k}$ as a subset of $B_{r}^{k+l}$ by $\left(t_{1}, \cdots, t_{k}\right) \rightarrow\left(t_{1}, \cdots, t_{k}, 0 \cdots 0\right)$. By a differentiable $k$-cell in $M$ we mean a one-to-one map $\phi: B_{1}^{k} \rightarrow M$ such that for each $t \in B_{1}^{k}$ there is a neighborhood $U$ of $t$ in $R^{k}$ and a nonsingular differentiable map $f: U \rightarrow M$ such that $f$ and $\phi$ agree on $U \cap B_{1}^{k}$. It follows from well-known extension theorems that if $\epsilon>0$ is sufficiently small then $\phi$ can be extended to a one-to-one nonsingular differentiable map of $\dot{B}_{1+\epsilon}^{k}$ into $M$. We will write $|\phi|$ for the image of $\phi$. We note that a differentiable $n$-cell $\phi$ in $M$ determines a coordinate system in $|\phi|$, and hence an orientation of $M$ if $M$ is orientable.

2. Extending a $k$-cell to an $n$-cell. The theorem of this section ex-

Received by the editors June 3, 1959. 